\title{
Degradation of Organic Pollutants by Titanium Dioxide Matrix Composites
}

\author{
Li Han ${ }^{1, a^{*}}$, Yan Gao ${ }^{1, b}$, Xing Gao ${ }^{1, \mathrm{c}}$, Weijuan Gong ${ }^{1, \mathrm{~d}}$, Hui Wang ${ }^{1, \mathrm{e}}$ \\ ${ }^{1}$ School of Chemical and Biological Engineering, Qilu Institute of Technology, Jinan, Shandong, 250200, China
}

\begin{abstract}
As a semiconductor material, titanium dioxide has the advantages of stable chemical properties, no pollution and low price, which is widely used in the field of organic pollutant degradation. The modification methods of semiconductor composite and nonmetallic doping for titanium dioxide are described. The degradation efficiency of modified titanium dioxide composite for organic pollutants is proposed. The advantages and disadvantages of the composite in preparation and application are pointed out. Finally, the development direction of modified titanium dioxide composite in the future is described.
\end{abstract}

\section{Introduction}

With the development of science and technology, the environmental pollution is increasing. Among them, the longest residual time, the most toxic organic pollutants. It is found that the areas with the fastest industrial development also have the most serious organic pollution. Organic pollutants mainly exist in water bodies in the form of polychlorinated biphenyls, halogenated hydrocarbons and polycyclic aromatic hydrocarbons, which affect human growth and development, nervous system, immune system and endocrine system, and increase the incidence of cancer. The treatment methods of organic pollution mainly include physical method, microbial method and chemical method. Among them, the microbiological method is widely used in the treatment of sewage and has a better effect. However, with the acceleration of society and modern industrial process, organic pollution types increase, microbial method has been unable to effectively remove organic pollutants, so it is necessary to find a clean and effective method of degradation of organic pollutants.

Photocatalytic technology utilizes the photocatalytic performance of semiconductor to dissolve organic pollutants in wastewater under illumination and achieve the effect of environmental treatment [1].

Since 1972, Japanese scientist Fujishima etal. discovered that titanium dioxide can decompose water to produce hydrogen under light, titanium dioxide as a photocatalyst has attracted the attention of scientists [2]. Titanium dioxide is widely used in the degradation of various organic pollutants in the environment because of its advantages of low cost, non-toxicity, high efficiency and high stability. However, the photocatalytic performance of $\mathrm{TiO}_{2}$ is limited due to its small visible light absorption range and high recombination rate of photogenerated electrons and holes. Studies have shown that $\mathrm{TiO}_{2}$ modified by ion doping and semiconductor composite has a wider range of light absorption, and its photocatalytic performance is further improved.

In this paper, the degradation performance of graphene oxide, carbon, nitrogen and cadmium sulfide doped modified titanium dioxide composites for organic pollutants was reviewed.

\section{Degradation of organic pollutants by graphene-titanium dioxide composites}

He etal. prepared $\mathrm{GO}-\mathrm{TiO}_{2}$ nanocomposites by ultrasonic-assisted method [3]. The results showed that the photodegradation efficiency of $\mathrm{GO}-\mathrm{TiO}_{2}$ nanocomposites was as high as $99 \%$, as shown in figure 1(a), and the photocatalytic activity of $\mathrm{GO}-\mathrm{TiO}_{2}$ nanocomposites remained high after recycling, as shown in figure 1(b). SEM and fluorescence analysis showed that GO still maintained its lamellar structure without obvious reaccumulation, and the prepared $\mathrm{GO}^{-\mathrm{TiO}_{2}}$ composite material had uniform dispersion, as shown in figure 2 , indicating that the ultrasonic-assisted method could maintain the structural integrity of GO to a certain extent. 

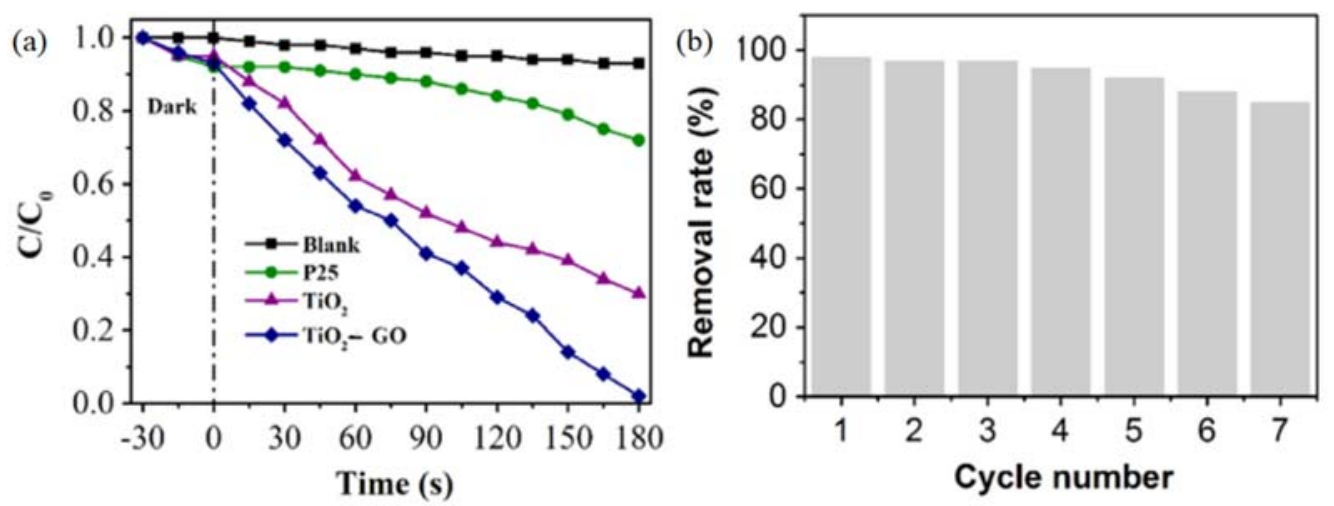

Figure 1 (a) Degradation characteristics of rhodamine B by different photocatalysts under visible light irradiation (b) Recycling of photocatalyst.
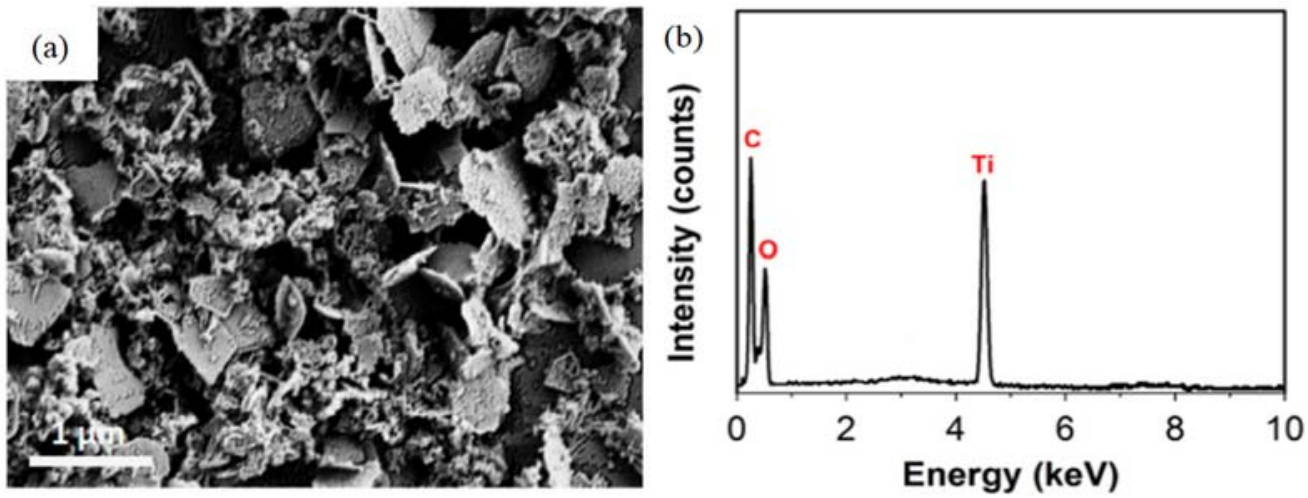

Figure 2 (a) Scanning electron microscopy of $\mathrm{GO}_{-} \mathrm{TiO}_{2}$ (b) Graphene oxide-titanium dioxide fluorescence analysis.

\section{Degradation of organic pollutants by carbon and nitrogen doped titanium dioxide composites}

\subsection{Carbon doped $\mathrm{TiO}_{2}$}

Lu prepared carbon dioxide materials with different roasting temperatures by sol method, using butyl titanate as titanium source and glucose as carbon source, and investigated their photodegradation performance of rhodamine $B$ [4]. The results showed that the degradation efficiency of rhodamine $B$ reached the highest when the roasting temperature was $500^{\circ} \mathrm{C}$ in the nitrogen atmosphere, reaching $61 \%$, as shown in figure 3(a). According to UV-Vis characterization, the reason is that its band gap width is reduced, which is the key to improve the photocatalytic degradation of organic pollutants, as shown in figure $3(\mathrm{~b})$.
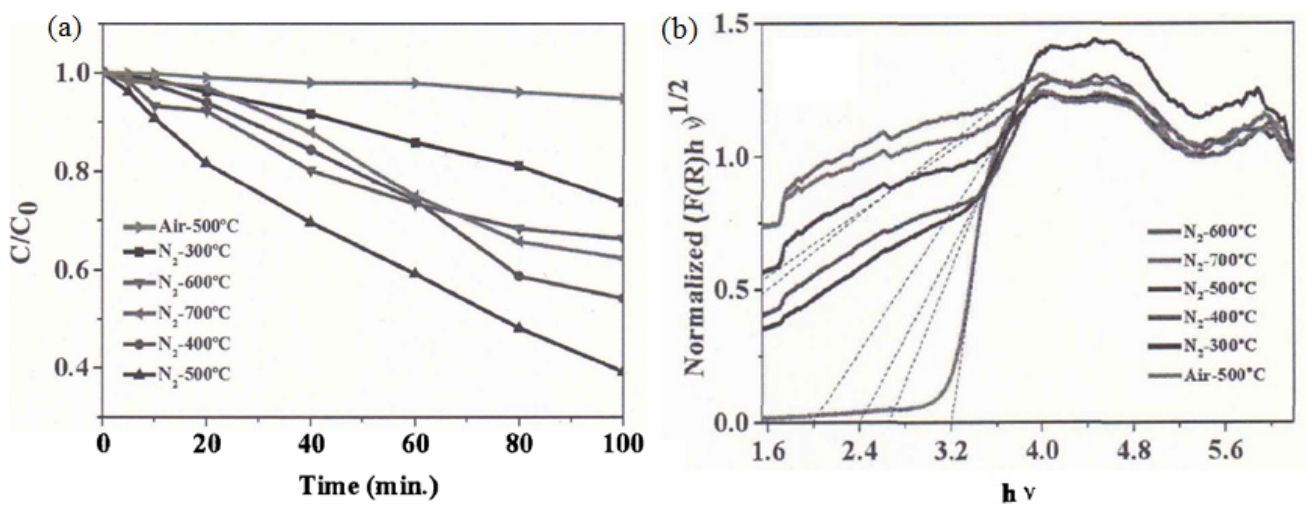

Figure 3 (a) Visible light photocatalytic performance of samples calcined at different temperatures in high purity nitrogen (b) UV-Vis diffuse reflection and absorption spectra of the samples prepared under different calcination conditions. 


\subsection{Nitrogen doped $\mathrm{TiO}_{2}$}

Zhang etal. prepared $\mathrm{TiO}_{2}$ composites with different nitrogen doping amount by hydrothermal method, and investigated their photocatalytic degradation performance of rhodamine $B$ [5]. The results show that when the nitrogen doping amount is $1 \%$, the degradation efficiency of rhodamine $B$ reaches the highest, reaching $98 \%$, as shown in figure 4 . The reason is that nitrogen-doped titanium dioxide reduces the band gap of titanium dioxide and expands the absorption range of light, thus improving the photocatalytic activity.

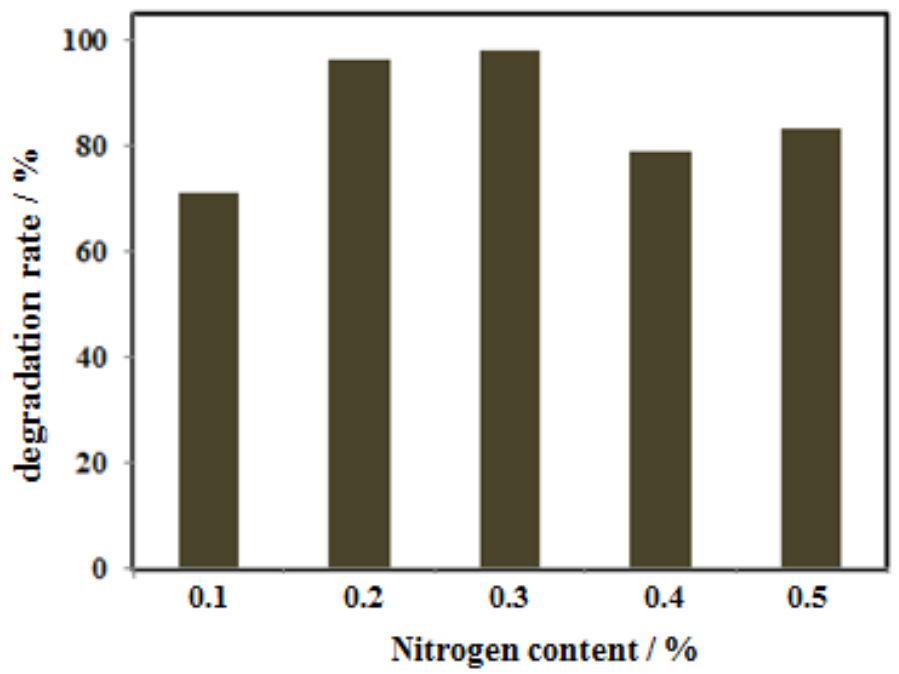

Figure 4 Degradation efficiency of rhodamine B by titanium dioxide with different nitrogen doping amounts

At present, the degradation mechanism of nitrogen-titanium dioxide on organic pollutants is still in a theoretical state, and the specific form of doping is still not clear, which still needs to be further studied.

\section{Degradation of organic pollutants by CdS-TiO2 composites}

Cheng etal. used anatase type titanium dioxide as titanium source to prepare $\mathrm{CdS} /$ titanium dioxide composite material by water bath method, and investigated its degradation performance of rhodamine $B$ [6]. The results showed that when $\mathrm{n}(\mathrm{CdS}) / \mathrm{n}\left(\mathrm{TiO}_{2}\right)=0.1$, the degradation efficiency of romintan $\mathrm{B}$ was as high as $91.9 \%$, as shown in figure 5 . According to the characterization of electrochemical resistance spectroscopy (EIS), the reason is that $\mathrm{CdS}_{2}-\mathrm{TiO}_{2}$ has a small arc, which generates charge transfer on the arc surface. The smaller the arc is, the greater the separation probability of photogenerated electrons and holes is, as shown in figure 6(a). In addition, Mott-Schottky measurements show that the doped $\mathrm{TiO}_{2}$ has a lower band gap relative to $\mathrm{TiO}_{2}$, as shown in figure $6(b)$.

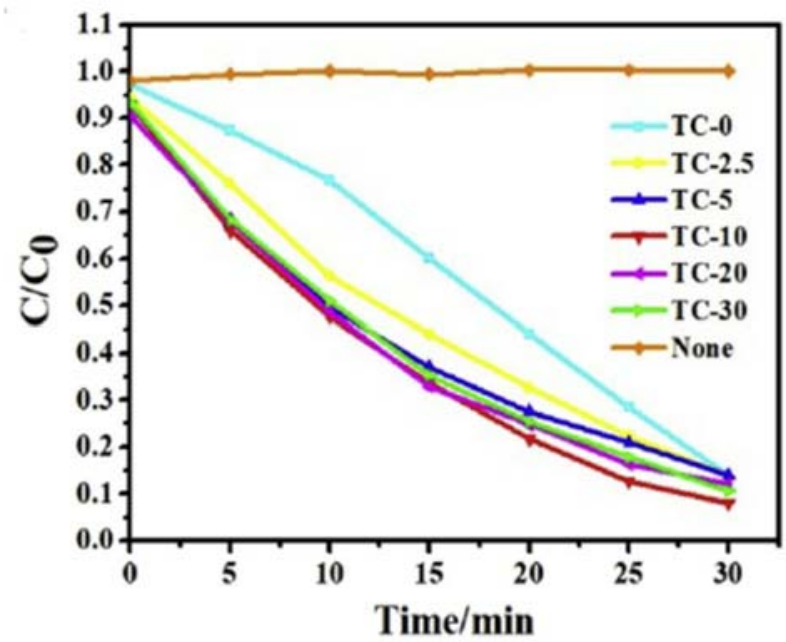

Figure 5 Degradation of rhodamine B in the presence of the original sample. 

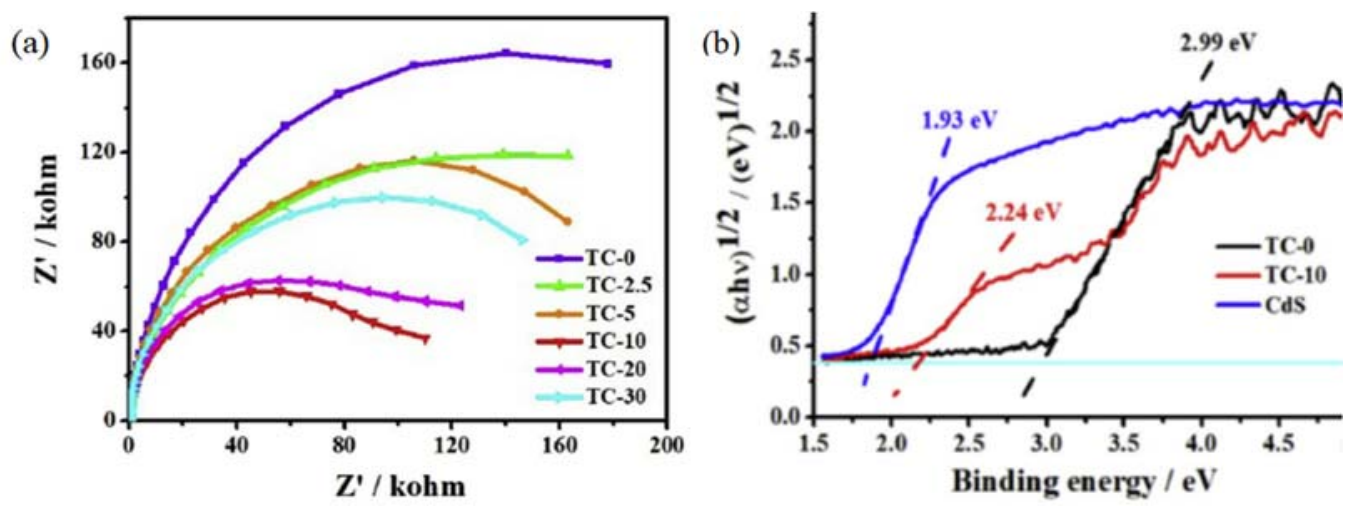

Figure 6 (a) Electrochemical resistance spectra of the prepared samples (b) $\mathrm{CdS} \backslash \mathrm{TiO}_{2}, \mathrm{CdS}-\mathrm{TiO}_{2} \mathrm{Mott}_{-} \mathrm{Schottky}$ diagram.

Although the hydrothermal synthesis of CdS- $\mathrm{TiO}_{2}$ can improve the photocatalytic activity, the preparation of $\mathrm{CdS}$ still has some problems such as high reaction conditions, poor crystallinity and hard preservation.

\section{Conclusion and prospect}

Through the review found that titanium dioxide composite material can effectively improve the efficiency of organic pollutants degradation, reach the role of purifying environment. The graphene oxide, titanium dioxide composite material, can effectively reduce the titanium dioxide forbidden band width, increase the range of light absorption, thus improve the photocatalytic activity. But in the process of the preparation of composite materials, graphene oxide structure easily destroyed, not very well to create synergies. Carbon and nitrogen non-metal doped titanium dioxide to obtain the composite material, due to the doping of non-metal, resulting in titanium dioxide lattice defects, the formation of a new impurity level, increase the titanium dioxide light response region, improve the efficiency of photocatalytic degradation. After the composite of $\mathrm{CdS}$ and $\mathrm{TiO}_{2}$ semiconductor, a new energy level difference will be formed between the semiconductor, which prevents the composite of photogenerated electron and hole, and improves the photocatalytic degradation efficiency of $\mathrm{TiO}_{2}$ for organic pollutants.

At present, the future development direction is to further study the structure and photocatalytic mechanism of titanium dioxide composite materials, so that it can give full play to its photocatalytic activity when degrading organic pollutants, completely degrading organic pollutants into water, carbon dioxide and small molecule inorganic substances, and effectively solving the problem of environmental pollution.

\section{References}

1 Luo L.J., Wang J., Pan X.J., etal. (2013) Titanium dioxide selective photocatalytic degradation of organic pollutants is reviewed. J. chemistry, 76: 332-337.

2 Fujishima A., Honda K. Electrochemical photolysis of water at a semiconductor electrode[J]. Nature, 1972, 238(5358): 37-38

3 He R., He W. (2016) Ultrasonic assisted synthesis of $\mathrm{TiO}_{2}$-reduced graphene oxide nanocomposites with superior photovoltaic and photocatalytic activities. J. Ceramics International, 42: 5766-5771.

4 Lu B. (2017) Carbon doping and organic molecules modified $\mathrm{TiO}_{2}$ nanoparticles preparation, characterization and application research. D. Hangzhou Normal University.

5 Zhang Y., Li L.H., Yan Y. (2015) Nitrogen doped $\mathrm{TiO}_{2}$ visible light catalytic degradation of nitrogen pollution research. J. Dali college courtyard, 14: 51-55.

6 Cheng X.H., Shang Y.R., Cui Y.P., etal. (2019) Enhanced photoelectrochemical and photocatalytic properties ofanatase- $\mathrm{TiO}_{2}(\mathrm{~B})$ nanobelts decorated with CdS nanoparticles. J. Solid Satate Sciences, 99: 106075 . 